\title{
Molecular characterization and differential expression of cytokinin-responsive type-A response regulators in rice (Oryza
} sativa)

\author{
Mukesh Jain, Akhilesh K Tyagi and Jitendra P Khurana*
}

Address: Interdisciplinary Centre for Plant Genomics and Department of Plant Molecular Biology, University of Delhi South Campus, Benito Juarez Road, New Delhi-110021, India

Email: Mukesh Jain - mjainanid@gmail.com; Akhilesh K Tyagi - akhilesh@genomeindia.org; Jitendra P Khurana* - khuranaj@genomeindia.org

* Corresponding author

Published: I3 February 2006

BMC Plant Biology 2006, 6:1 doi:10.1 186/147/-2229-6-1
Received: 16 August 2005

Accepted: 13 February 2006

This article is available from: http://www.biomedcentral.com/I47/-2229/6/I

(c) 2006 Jain et al; licensee BioMed Central Ltd.

This is an Open Access article distributed under the terms of the Creative Commons Attribution License (http://creativecommons.org/licenses/by/2.0), which permits unrestricted use, distribution, and reproduction in any medium, provided the original work is properly cited.

\begin{abstract}
Background: The response regulators represent the elements of bacterial two-component system and have been characterized from dicot plants like Arabidopsis but little information is available on the monocots, including the cereal crops. The aim of this study was to characterize type-A response regulator genes from rice, and to investigate their expression in various organs as well as in response to different hormones, including cytokinin, and environmental stimuli.
\end{abstract}

Results: By analysis of the whole genome sequence of rice, we have identified ten genes encoding type-A response regulators based upon their high sequence identity within the receiver domain. The exon-intron organization, intron-phasing as well as chromosomal location of all the RT-PCR amplified rice (Oryza sativa) response regulator (OsRR) genes have been analyzed. The transcripts of OsRR genes could be detected by real-time PCR in all organs of the light- and dark-grown rice seedlings/plants, although there were quantitative differences. The steady-state transcript levels of most of the OsRR genes increased rapidly (within $15 \mathrm{~min}$ ) on exogenous cytokinin application even in the presence of cycloheximide. Moreover, the expression of the OsRR6 gene was enhanced in rice seedlings exposed to salinity, dehydration and low temperature stress.

Conclusion: Ten type-A response regulator genes identified in rice, the model monocot plant, show overlapping/differential expression patterns in various organs and in response to light. The induction of $O s R R$ genes by cytokinin even in the absence of de novo protein synthesis qualifies them to be primary cytokinin response genes. The induction of OsRR6 in response to different environmental stimuli indicates its role in cross-talk between abiotic stress and cytokinin signaling. These results provide a foundation for further investigations on specific as well as overlapping cellular functions of type-A response regulators in rice.

\section{Background}

Cytokinins regulate various plant growth and developmental processes, including cell division, apical dominance, chloroplast biogenesis, leaf senescence, vascular differentiation, photomorphogenic development, shoot differentiation in tissue cultures and anthocyanin production, primarily by altering the expression of diverse genes $[1,2]$. The recent genetic and molecular studies in plants 
Table I: Type-A response regulators in rice.

\begin{tabular}{|c|c|c|c|c|c|c|c|c|c|}
\hline \multirow{2}{*}{$\begin{array}{l}\text { Gene } \\
\text { name }^{a}\end{array}$} & \multirow{2}{*}{$\begin{array}{c}\text { Accession } \\
\text { no.b }\end{array}$} & \multirow{2}{*}{$\begin{array}{c}\text { ORF } \\
\text { Length } \\
\text { (bp)c }\end{array}$} & \multirow{2}{*}{$\begin{array}{l}\text { Polypeptide } \\
\text { length }(\mathrm{aa})^{\mathrm{d}}\end{array}$} & \multirow{2}{*}{$\begin{array}{c}\text { No. of } \\
\text { introns }\end{array}$} & \multirow{2}{*}{$\begin{array}{c}\text { Chromosome } \\
\text { no. }\end{array}$} & \multicolumn{3}{|c|}{ Genomic locusg } & \multirow{2}{*}{$\begin{array}{l}\text { Nearest } \\
\text { markerh }\end{array}$} \\
\hline & & & & & & $\begin{array}{c}\text { BAC/PAC } \\
\text { name }\end{array}$ & $\begin{array}{c}\text { Accession } \\
\text { no. }\end{array}$ & $\begin{array}{c}\text { CM } \\
\text { position }\end{array}$ & \\
\hline OsRRI & A) 938070 & 696 & 231 & 5 & 4 & P0076017 & BX548156 & 65.3 & E60696S \\
\hline OsRR2 & AJ938071 & 759 & 252 & 5 & 2 & OSJNBb0038F20 & AP005808 & $81.7-83.6$ & C499 \\
\hline OsRR3 & A) 938072 & 396 & 131 & 3 & 2 & OJII24_D06 & AP004043 & 157.9 & $\mathrm{Cl} 470$ \\
\hline OsRR4 & A) 938073 & 699 & 232 & 4 & I & P043IG06 & AP003683 & 169.5 & SI058I \\
\hline OsRR5 & A) 938074 & 405 & 134 & 4 & 4 & OSJNBb0065J09 & $\overline{\mathrm{AL} 663010}$ & $78.2-81.7$ & CI558S \\
\hline OsRR6 & A] 938075 & 513 & 170 & 1 & 4 & OSJNBb0004AI7 & AL606652 & 123.8 & R223I \\
\hline OsRR7 & Aj938076 & 621 & 206 & 4 & 7 & OJ1047_A06 & AP003802 & 53.4 & C735 \\
\hline OsRR8 & A) 938077 & 366 & 121 & 4 & 8 & OJ1705_C03 & AP003962 & $60.1-60.4$ & E61839S \\
\hline OsRR9 & A) 938078 & 606 & 201 & 4 & 11 & OSJNBa0059J06 & $\mathrm{ACl} 23526$ & $8.1-8.6$ & R642A \\
\hline OsRRIO & A) 938078 & 606 & 201 & 4 & 12 & OSJNBb0034E23 & AL928752 & 12.2 & $\mathrm{R} 642 \mathrm{~B}$ \\
\hline
\end{tabular}

a Systematic designation given to rice type-A response regulator genes. ${ }^{\mathrm{b}} \mathrm{cDNA}$ Genbank accession number (OsRR9 and 10 has been assigned the same accession number as they could not be amplified specifically). c Length of open reading frame in base pairs. ${ }^{d}$ Length (number of amino acids) of the deduced polypeptide. e Number of introns present within ORF. $\mathrm{f}$ Chromosomal localization of OsRR gene. 8 Name, accession number and approximate $\mathrm{cM}$ position of the BAC/PAC clone in which the corresponding OsRR gene is present. h Nearest marker to the OsRR gene.

have suggested the involvement of two-component sensor-regulator system in cytokinin signal perception and transduction, comprising sensor histidine kinase (HK) proteins, histidine phosphotransfer (HPt) proteins, and effector response regulator (RR) proteins [3-9]. Such signal transduction systems, once thought to be restricted to prokaryotes, have also been found in many eukaryotes, including yeast, fungi, slime molds and higher plants [10]. In Arabidopsis, proteins with homology to all the elements of two-component system have been identified [7].

The analysis of Arabidopsis genome revealed the existence of 32 putative response regulator genes [7]. Based on the predicted protein domain architecture and amino acid composition, the response regulators have been broadly categorized into three distinct families: type-A, type-B and pseudo-response regulators. The type-A response regulators are relatively small, containing a receiver domain along with small $\mathrm{N}$ - and C-terminal extensions [11]. The type- $\mathrm{B}$ response regulators comprise a receiver domain fused to the DNA-binding domain and are supposed to be transcriptional regulators [12-14]. The pseudo-response regulators share significant sequence similarity with the receiver domain of other response regulators but the invariant D-D-K motif is not present [7]. The pseudoresponse regulators are also considered to be the elements of the circadian clock in Arabidopsis and rice [15-18].

The type-A response regulator genes in Arabidopsis (type-A $A R R s$ ) are rapidly and specifically induced by exogenous cytokinin, although with varying kinetics, and have been characterized as primary cytokinin response genes $[11,19,20]$. The transcription of type-A ARR genes is regulated in part by type-B ARRs $[21,22]$. Some of the type-A ARRs perform partially redundant functions, acting as negative regulators of cytokinin responses by a feedback mechanism $[21,23,24]$. In contrast, ARR4 was claimed to be a positive regulator of cytokinin signaling because its over-expression enhanced the cytokinin responsiveness of transgenic Arabidopsis plants [25]. However, the loss-offunction mutant did not reveal a positive role for ARR4 in cytokinin signaling [24] and this discrepancy remains to be resolved. The tissue distribution of ARR4 overlaps to a large extent with that of phytochrome B (phyB) and it has been found to interact with N-terminus of phyB to stabilize its active form [26]. The transgenic Arabidopsis plants overexpressing ARR4 are specifically hypersensitive to red light [26], indicating that ARR4 may be involved in integrating red light and cytokinin signaling.

The type-A response regulators have been isolated and characterized from maize $[27,28]$. However, there is no report on the characterization of any type-A response regulator from other monocot species, although several EST/ cDNA sequences are available in the databases. Here, we report the identification and analysis of type-A response regulator gene family in rice (Oryza sativa), the model monocot plant. The exon-intron organization, chromosomal distribution and sequence homology have been analyzed for all ten members. The OsRR genes express differentially in various organs examined, and also in response to light. The application of exogenous cytokinin 

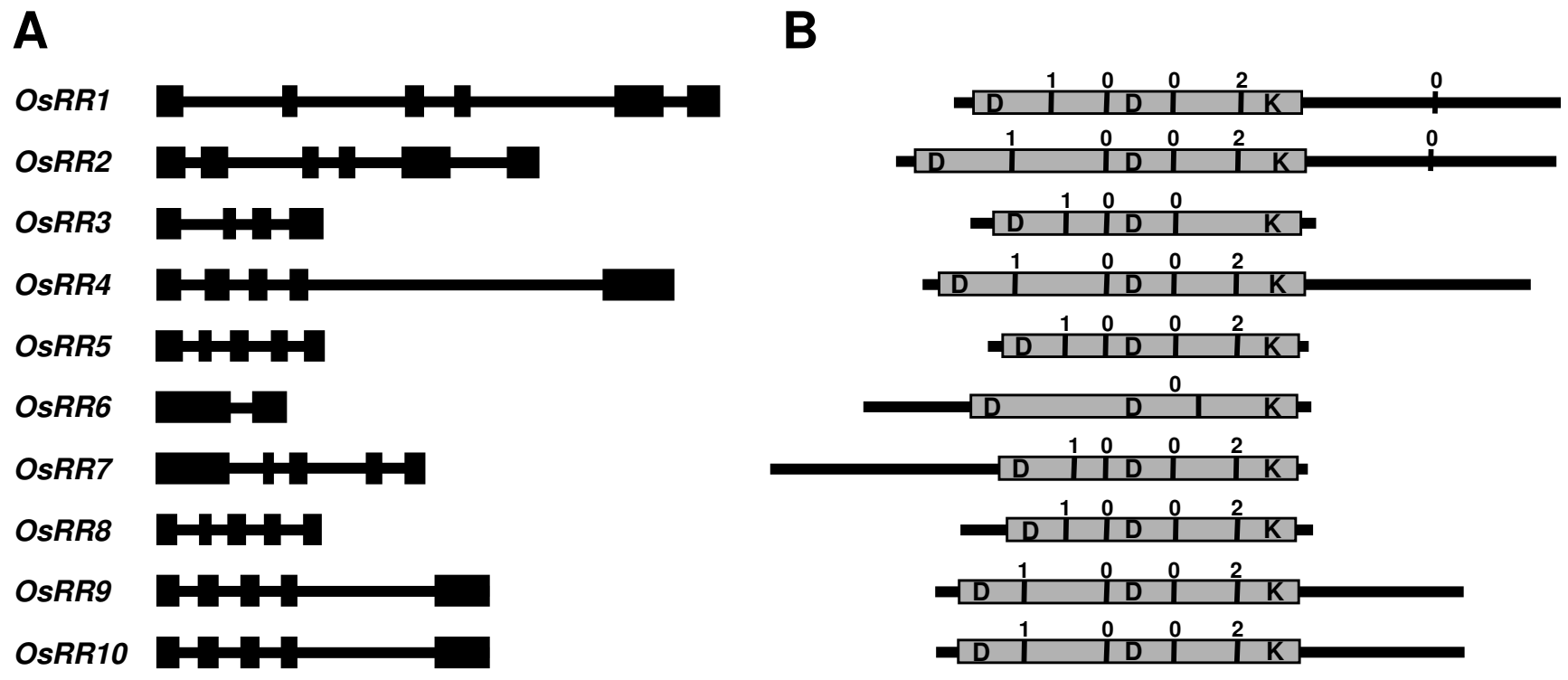

C

OsRR1
OsRR2
OsRR3
OsRR4
OsRR5
OsRR6
OsRR7
OsRR8
OsRR9
OsRR10

OsRR1
OsRR2
OsRR3
OsRR4
OsRR5
OsRR6
OsRR7
OsRR8
OsRR9
OsRR10

OsRR1
OsRR2
OsRR3
OsRR4
OsRR5
OsRR6
OsRR7
OsRR8
OsRR9
OsRR10

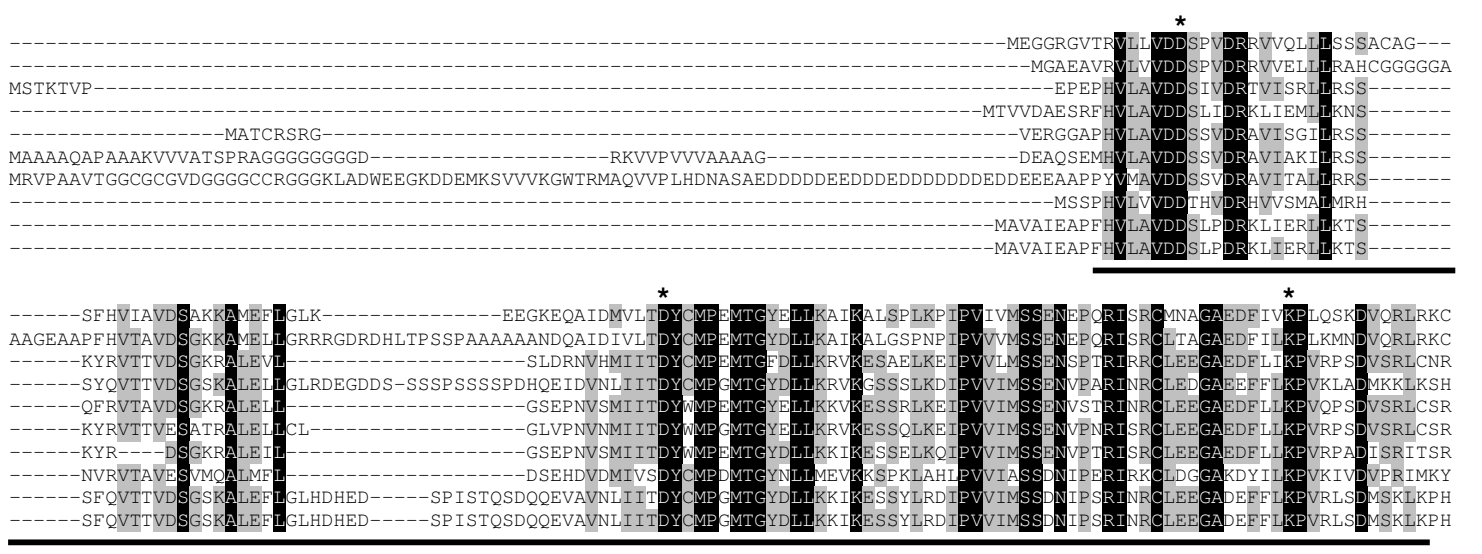

SPAN-TQCCDAGSDGKPPLLLLPSDHVVVDATAASPPPPPSRRR-AHFAGVAMVLHSSSVELSHYFPFLFKF ILLVYAI LCLGELLHRWSNG-CE LNLWCA SGATRP KSAVAGDDDR----CCNTAKKAAAAAAATP EQQQQQQRSSHLAGLAMVMNASSFEVSHYFQLIF KLILLAYAVLCLSQLLHRWSNGSSLLSLWCA VIMK--

LLKRKQLPMAAAAP DKPPHKPDEAAASAAA IAEAATAQTDGI ISDCSCSGSS KRKAAAMEQEVISSPDQRTKPRLSSTSSGLAVETVLR-

IR-

MLQ-

ILKSR-

ILKSR--

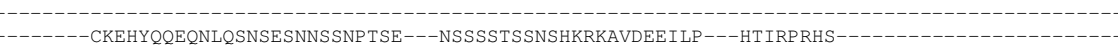

CKEHYQQEQNLQSNSESNNSSNP TSE---NSSSSTSSNSHKRKAVDEEILP---HTIRPRHS--

\section{Figure I}

Type-A response regulators in rice. (A) Exon-intron organization of OsRR genes. Exons and introns are represented by black boxes and lines, respectively. (B) Schematic representation of OsRR proteins (aligned with respect to the second conserved Asp (D) residue). The conserved receiver domain is represented as gray box with D-D-K residues. The black vertical bars represent intron position. The numbers $0, I$ and 2 above the vertical bars represent the phase $0, I$ and 2 introns, respectively. (C) Multiple alignments of the OsRR proteins obtained with ClustalX. Fully and partially conserved (present in more than $50 \%$ of aligned sequences) residues are highlighted in black and gray boxes, respectively. Gaps (marked with dashes) have been introduced to maximize the alignments. The conserved receiver domain has been underlined. Conserved Asp and Lys residues are marked with asterisks. 
Table 2: Percentage identities among the rice type-A response regulators.

\begin{tabular}{|c|c|c|c|c|c|c|c|c|c|c|}
\hline & OsRRI & OsRR2 & OsRR3 & OsRR4 & OsRR5 & OsRR6 & OsRR7 & OsRR8 & OsRR9 & OsRRIO \\
\hline OsRRI & - & $63.2 / 53.7$ & $44.4 / 44.3$ & $33.6 / 28.6$ & $47.7 / 47.0$ & $36.1 / 35.3$ & $29.1 / 27.7$ & $34.4 / 38.8$ & $34.8 / 29.9$ & $34.8 / 29.9$ \\
\hline OsRR2 & & - & $43.9 / 45.0$ & $35.9 / 32.3$ & $47.7 / 45.5$ & $40.2 / 30.6$ & $34.8 / 26.7$ & $36.6 / 38.8$ & $38.8 / 29.4$ & $38.8 / 29.4$ \\
\hline OsRR3 & & & - & $53.5 / 55.7$ & $66.7 / 71.0$ & $64.6 / 67.9$ & $60.6 / 62.6$ & $44.8 / 48.8$ & $51.3 / 55.0$ & $51.3 / 55.0$ \\
\hline OsRR4 & & & & - & $57.0 / 56.0$ & $41.5 / 45.3$ & $32.2 / 29.1$ & $46.7 / 44.6$ & $58.1 / 55.7$ & $58.1 / 55.7$ \\
\hline OsRR5 & & & & & - & $67.4 / 72.4$ & $67.4 / 68.7$ & $50.0 / 51.2$ & $54.3 / 55.2$ & $54.3 / 55.2$ \\
\hline OsRR6 & & & & & & - & $55.8 / 53.5$ & $38.5 / 47.9$ & $42.5 / 43.5$ & $42.5 / 43.5$ \\
\hline OsRR7 & & & & & & & - & $44.3 / 43.8$ & $31.8 / 29.4$ & $31.8 / 29.4$ \\
\hline OsRR8 & & & & & & & & - & $43.7 / 43.8$ & $43.7 / 43.8$ \\
\hline OsRR9 & & & & & & & & & - & $99.4 / 100.0$ \\
\hline OsRRIO & & & & & & & & & & - \\
\hline
\end{tabular}

Values indicate the percentage identity obtained by pairwise comparisons between ORF (left) and protein sequences (right).

induced $O s R R$ genes in the absence of de novo protein synthesis. Evidence has also been provided for a probable role of OsRR6 in abiotic stress signaling.

\section{Results and discussion}

\section{Identification of type-A response regulators in rice}

The information on rice genomic sequence $[29,30]$ provides a powerful tool to identify putative homologous proteins by database searches with genes of known function from other organisms. In an attempt to identify typeA response regulators in rice, the whole rice genome, dynamically translated in all reading frames, was analyzed employing TBLASTN search, using Arabidopsis type-A response regulator proteins as query. This search identified a total of ten genes showing high sequence similarity with the Arabidopsis type-A response regulators. These predicted proteins contain a characteristic receiver domain (harboring conserved D-D-K residues) with short $\mathrm{N}$ - and C-terminal extensions and were designated as Oryza sativa response regulator (OsRR) proteins. We amplified (by RTPCR) and sequenced nine OsRR cDNAs (OsRR9 and 10 could not be amplified specifically since they possess $>99 \%$ identity within the coding region and flanking sequences). Their sequences were confirmed by comparison with the corresponding genomic sequences. The cDNA sequences have been deposited in the GenBank, and their accession numbers, predicted protein length and genomic locus are given in Table 1.

\section{Exon-intron organization and chromosomal distribution}

A comparison of the full-length cDNA sequences (obtained in the present study) with the corresponding genomic DNA sequences (available in the database) showed that the coding sequence of majority of the OsRR genes ( 6 out of 10 ) are disrupted by 4 introns (Table 1 , Fig. 1A), suggesting their origin from a common ancestral gene with a classical pattern of 5 exons and 4 introns. However, variations in their basic gene structure were observed for other members, implicating gain (OsRR1 and OsRR2) or loss (OsRR3 and OsRR6) of introns. The highly conserved intron phasing and the position of introns with respect to their amino acid sequences (Fig. $1 \mathrm{~B})$ also indicate their evolution from the same ancestral gene by exon shuffling [31].

The BAC (bacterial artificial chromosome) or PAC (phage artificial chromosome) clones carrying the genes for OsRR proteins were identified (Table 1). The chromosome map positions of BAC/PACs given in centiMorgans (cM) from top of the chromosome, and the nearest marker to each OsRR gene are indicated in Table 1. The ten OsRR genes were found to be distributed on 7 of the 12 rice chromosomes (Table 1). Three OsRR genes are present on chromosome 4, two on chromosome 2 , and one each on

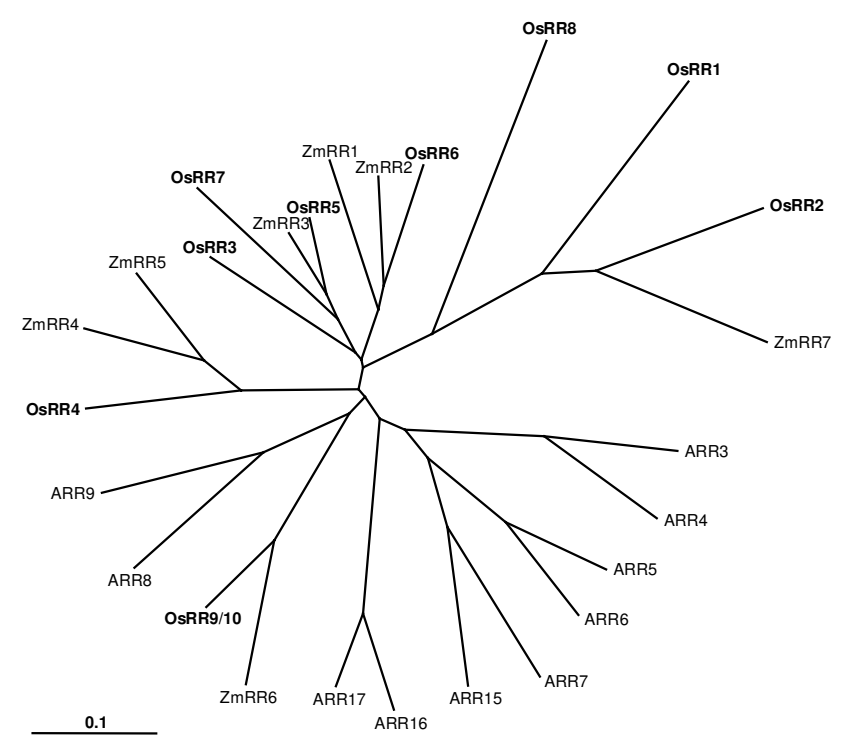

Figure 2

Phylogenetic relationship among type-A response regulator proteins from rice (OsRR), maize (ZmRR), and Arabidopsis (ARR). The unrooted tree was generated using ClustalX program by neighbor-joining method and visualized by Treeview. Scale bar represents 0.1 amino acid substitution per site. 


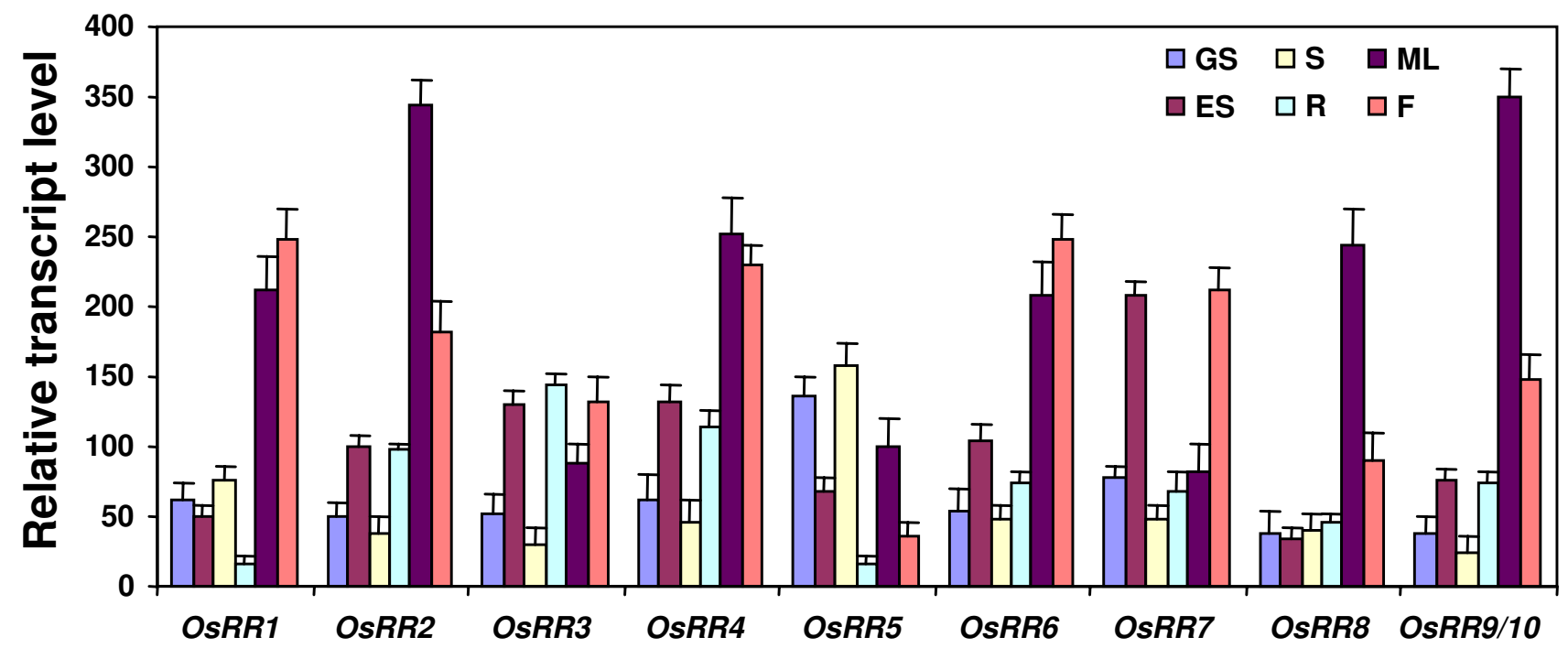

Figure 3

Real-time PCR analysis showing the organ-specific expression profiles of individual OsRR genes. The relative mRNA levels of individual OsRR genes normalized with respect to housekeeping gene, UBQ5, in different tissues (GS, green seedlings; ES, etiolated seedlings; S, green shoots; R, roots; $M L$, mature leaves; F, flowers) are presented.

chromosome $1,7,8,11$, and 12 . The distribution of OsRR genes on rice chromosomes did not reveal evident clusters. However, OsRR9 and OsRR10 are present on the duplicated block between chromosome 11 and 12 [32,33].

\section{Sequence analysis}

The type-A RRs are mainly composed of a receiver domain with short N- and C-terminal extensions [34], essentially similar to the $E$. coli response regulator (RR) CheY involved in chemotaxis, and lack a typical output domain. All the OsRR proteins also contain the highly conserved Lys and two Asp residues (D-D-K) in the receiver domain (Fig. 1B, C). Pairwise analysis of the full-length OsRR protein sequences indicates that the overall identities range from $28 \%$ to $71 \%$ (100\% between OsRR9 and 10, Table 2 ). However, the amino acid identity within the receiver domain reaches up to $95 \%$. The receiver domain of typeA RRs in Arabidopsis also showed 60\% to 93\% identity among them but less than $30 \%$ with that of type-B RRs. The predicted amino acid sequences of $\mathrm{N}$ - and C-terminal extensions of OsRR proteins are more variable (Fig. 1C), like Arabidopsis RR proteins [11]. The C-terminal extensions of ARR6 and ARR7 were shown to be responsible for their nuclear localization [35]. The rice type-A response regulators, OsRR1, OsRR2, OsRR4, OsRR9 and OsRR10, also possess C-terminal extensions rich in acidic and charged residues (Fig. 1B,C). However, OsRR6 and OsRR7 have $\mathrm{N}$-terminal extensions rich in gly and asp residues (Fig. 1C). These N- and C-terminal variable regions may play a role in their localization to different cellular compartments.

To examine the phylogenetic relationships of rice, maize, and Arabidopsis type-A response regulators, an unrooted tree was constructed from alignments of their full-length protein sequences (Fig. 2). The OsRR proteins formed four sister pairs (OsRR1 and 2, OsRR3 and 6, OsRR5 and 7, OsRR9 and 10). This pairing of type-A RRs is consistent with recent evolutionary duplications postulated to have occurred in the rice genome [32,36]. The type-A response regulators in Arabidopsis also formed five sister pairs and all of them were found to be located on homologous duplicated chromosomal segments [24]. Thus, it is remarkable that the duplication of the sister pairs of typeA response regulator genes is associated with chromosomal block duplications in both rice and Arabidopsis. Recently, the extensive duplication and preferential retention of early auxin-responsive Aux/IAA genes have been reported in both Arabidopsis and rice [37,38]. The retention of the duplicated type-A response regulator genes in rice (present study) also supports the idea that the genes involved in transcription and signal transduction have been preferentially retained in Arabidopsis [39]. Also, most of the OsRRs clustered together alongwith maize RRs (ZmRRs), in clades distinct from Arabidopsis RRs (ARRs) (Fig. 2). In an earlier study with Arabidopsis RRs, a phylogenetic analysis, including only a few of the monocot RRs, revealed that the type-A ARRs fall into clades distinct from rice and maize [24]. The present study corroborates this 


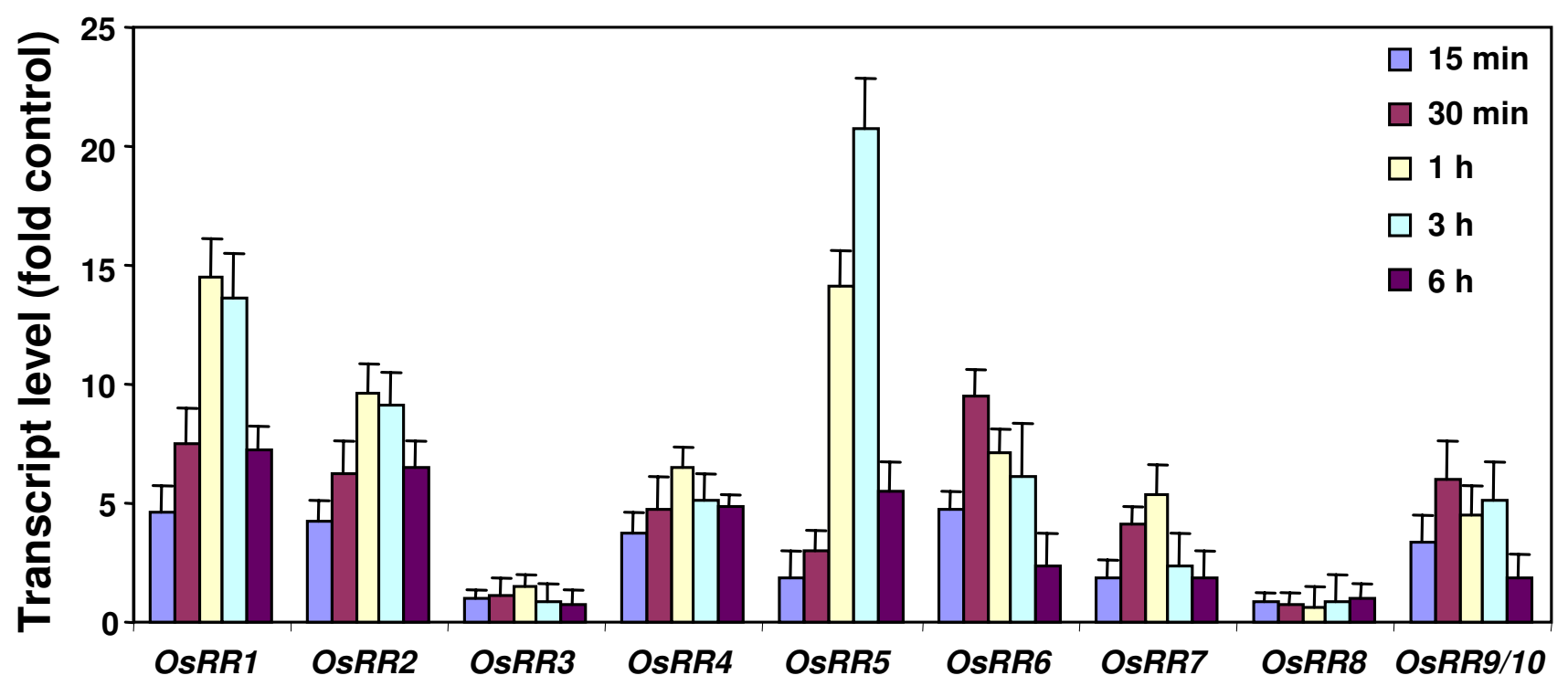

Figure 4

Kinetics of induction of OsRR genes in response to cytokinin. The transcript levels of each OsRR gene in 6-day-old light-grown seedlings treated with BAP for $15 \mathrm{~min}, 30 \mathrm{~min}, \mathrm{I} \mathrm{h}, 3 \mathrm{~h}$ and $6 \mathrm{~h}$, were plotted as the relative expression (fold) of the seedlings mock-treated for the same duration.

observation and strengthens the view that the progenitor of monocots and dicots probably had only a small family of RRs and the expansion occurred subsequently, in both monocots and dicots, by gene duplication.

\section{Organ-specific expression of OsRR genes}

To examine the expression pattern of each OsRR gene in different plant organs, and to assess the effect of light, quantitative real-time PCR analysis was performed with total RNA isolated from etiolated seedlings, green seedlings, green shoots, roots, mature leaves, and flowers. The transcripts of all the OsRR genes were detected in various organs examined but they displayed a complex expression pattern (Fig. 3). Most of the OsRR genes were expressed at relatively higher level in mature tissues (leaves and flowers). The expression of OsRR genes was also higher in roots (present study), which is essentially similar to most of the ARRs [11]. The transcript levels of OsRR2, 3, 4, 6, 7, and 9 were significantly higher in etiolated seedlings as compared to green seedlings (Fig. 3). However, OsRR5 exhibited higher expression in green seedlings. Interestingly, in a recent study, the arr mutants in Arabidopsis exhibited altered red light sensitivity [24]. Also, ARR4 has been found to interact with phytochrome $B$ and stabilize its active form [26]. These results suggest that OsRR genes exhibit overlapping and differential expression patterns, playing predominant roles in specific organs and may also be involved in light signal transduction.

\section{OsRRs represent primary cytokinin response genes}

Cytokinin regulates the expression of a large number of genes including type-A response regulators in Arabidopsis and maize $[11,27,40]$. To study the effect of cytokinin on the transcript levels of individual OsRR genes, the lightgrown rice seedlings were treated with exogenous cytokinin for various durations over a $6 \mathrm{~h}$ period, and real-time PCR analysis was performed. Consistent with the results reported earlier with Arabidopsis [11], the steady-state transcript levels of OsRR genes were elevated within $15 \mathrm{~min}$ of exogenous cytokinin application (Fig. 4). The kinetics of induction with cytokinin was similar for most OsRR genes, with the transcript levels reaching its maximum in $1 \mathrm{~h}$, and declining thereafter. There was, however, no significant change in the transcript abundance of OsRR3 and 8 following cytokinin treatment (Fig. 4). The rapid induction of type-A ARRs by exogenous cytokinin has been shown to mediate a feedback mechanism, which decreases the sensitivity of the plant to the hormone, indicating that type-A RRs act as negative regulators of cytokinin-induced responses $[21,23,24]$. The type-B RRs are the transcription factors that are involved in the transcriptional activation of the type-A response regulators in response to cytokinins $[13,21,22]$.

Many primary response genes, such as auxin-induced $A u x /$ IAA genes, are induced in the absence of de novo protein synthesis [41]. The genes encoding type-A RRs in Arabidop- 


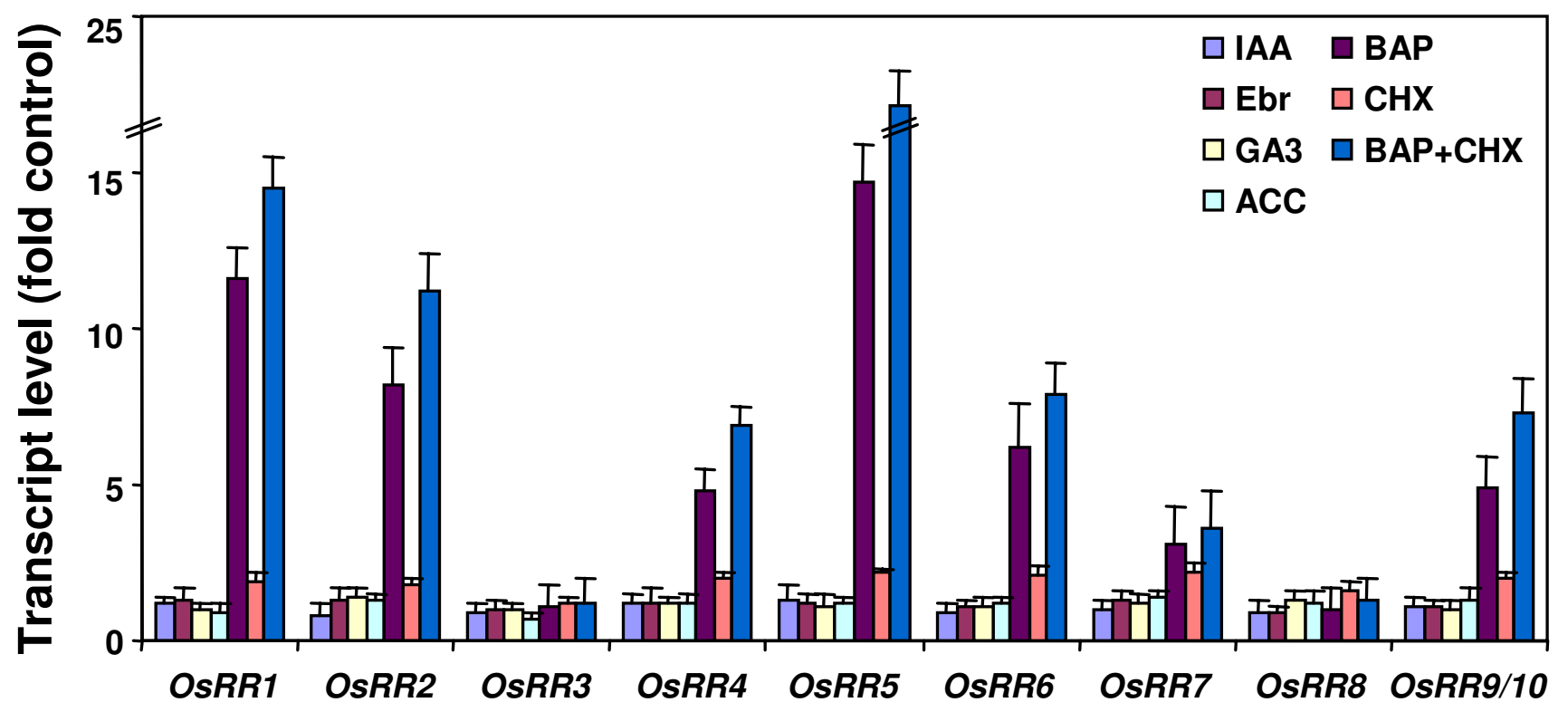

Figure 5

Expression profiles of the OsRR genes in response to different hormones and cycloheximide. The transcript levels of each OsRR gene in 6-day-old light-grown seedlings treated with IAA, Ebr, GA3, ACC, BAP, CHX and CHX+BAP for $3 \mathrm{~h}$, were plotted as the relative expression (fold) of the seedlings mock-treated for the same duration.

sis have also been described as the primary cytokinin response genes $[11,42]$. To determine whether the OsRR genes also belong to the same category, their induction by cytokinin was examined in the presence of a protein synthesis inhibitor, cycloheximide (Fig. 5). The steady-state transcript levels of most of the OsRR genes increased (up to 2- to 2.5-fold) on treatment with cycloheximide, suggesting that the transcription of these genes is regulated by a short-lived repressor protein. The increase in transcript abundance (up to 4- to 6-fold) of rice $A u x / I A A$ genes examined (OSIAA9, OsIAA13 and OsIAA20) [38] in the same RNA samples (data not shown), confirmed that cycloheximide treatment was biologically effective in our experiments. Further, cycloheximide failed to block the induction of these genes by cytokinin; the transcript levels of OsRR genes were higher in the samples treated with BAP in the presence of cycloheximide as compared to BAP alone (Fig. 5). These results suggest that OsRR genes constitute a class of primary cytokinin response genes.

The expression of OsRR genes in response to other plant hormones, including auxin, brassinosteroid, gibberellin and ethylene, was also examined. However, no significant effect of these hormones could be detected on the steadystate transcript levels of OsRR genes (Fig. 5), indicating their role primarily in cytokinin signaling.

\section{Expression of OsRR genes under stress conditions}

It has been claimed earlier that the expression of some of the type-A response regulators in Arabidopsis is induced by different environmental stresses such as drought, salinity and low temperature [43]. To investigate, whether some of the OsRR genes are also involved in stress responses, their expression levels were examined by real-time PCR analysis of the RNA isolated from young rice seedlings subjected to various abiotic stress treatments. The expression of a majority of $O s R R$ genes was not significantly altered under stress (data not shown), with the notable exception of OsRR6. The expression of OsRR6 gene was induced to significant levels by salt, dehydration and low temperature treatments (Fig. 6), and results were reproducible. This indicates that OsRR6 may play an important role in abiotic stress signaling in rice, besides acting as a component in cytokinin signaling. It is noteworthy here that a transmembrane hybrid-type histidine kinase, AHK1, closely related to cytokinin receptors, is a putative osmosensor in Arabidopsis [44].

\section{What are the probable functions of OsRR proteins?}

The type-A RRs in Arabidopsis have been shown to act as the negative regulators of cytokinin signaling with partially redundant functions $[11,24]$. In addition, some of these ARRs and other elements of the two-component 


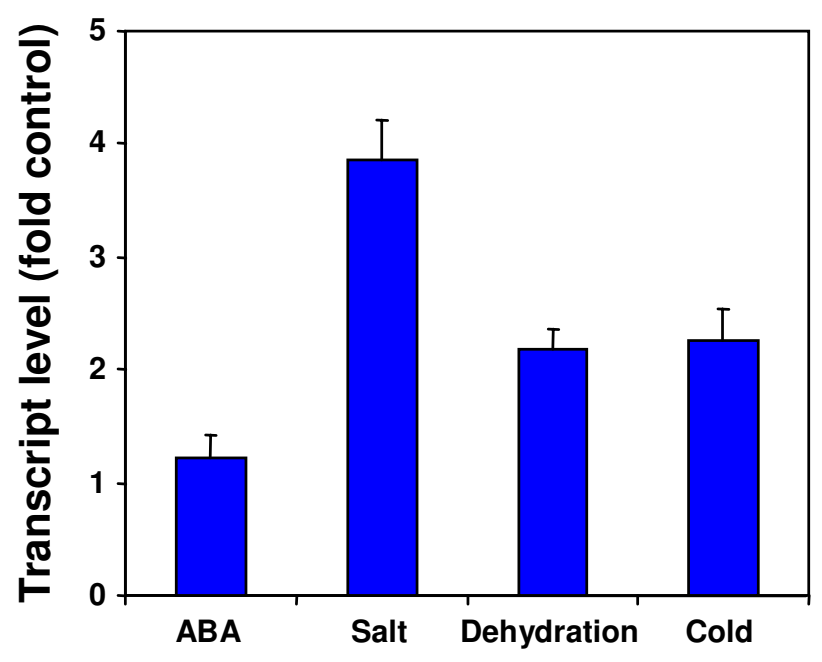

Figure 6

Changes in transcript levels of the OsRR6 gene in response to different stress treatments. The transcript levels of OsRR6 gene in 6-day-old light-grown seedlings treated with $A B A$, $\mathrm{NaCl}$, mannitol and cold for $6 \mathrm{~h}$, were plotted as the relative expression (fold) of the seedlings mock-treated for the same duration.

sensor-regulator phosphorelay participate in cross-talk between cytokinin and light signaling (mediated by phytochromes) and also with the gaseous hormone ethylene $[4,45]$. The role of one of the type-A RRs, i.e. ARR4, has also been ascribed in red light mediated photomorphogenesis $[24,26]$. In fact, ARR4 physically associates with phytochrome B to prolong the stability of its active conformation, Pfr, and accentuates red light signaling. The role of type-B ARRs (and possibly of type-A ARRs) has also been envisaged in a cross-talk between ethylene and cytokinin signaling, although they may regulate these components differentially to control diverse plant processes [4]. To have an inkling about the functions of OsRR genes, the rice Tos 17 insertion mutant database [46] providing the phenotype of rice Tos17 retrotransposon insertion mutants [47] was accessed using the BLAST program. We could identify insertion mutants corresponding only to OsRR9/10 gene (NE6006_0_401_1A and ND8005_0_402_1A); it was difficult to demarcate whether the sequence flanking the Tos 17 insertion represents OsRR9 or OsRR10 because of more than $99 \%$ similarity between them. Despite the fact that OsRR 9 and OsRR10 genes are so similar and may function redundantly, the phenotype of the insertion mutants (representing only one of these two loci) showed dwarfism, sterility, lesion mimic and vivipary. It can thus be speculated that OsRR9/10 genes may quantitatively affect different cellular processes influenced by both light and cytokinin. A detailed analysis of the insertional mutants already avail- able and RNAi strategy for the remaining OsRR genes will greatly help in elucidation of the precise role of these genes.

\section{Conclusion}

The results of structural analyses of rice type-A RR proteins and their phylogenetic relationship with ARRs will be helpful for their functional validation in rice. The organspecific differential expression profile of OsRR genes suggests that their products most likely perform diverse and overlapping functions in different cell types of rice. This study also reflects the role of OsRR genes in both light and cytokinin signaling. The induction of OsRR6 by different abiotic stress stimuli provides a molecular link between stress and cytokinin signaling as well. These results provide a foundation for future work on the elucidation of cellular functions of type-A response regulators in rice.

\section{Methods}

\section{Sequence analysis}

The type-A response regulator genes in rice were identified by performing BLAST searches at the National Centre for Biotechnology Information [48] and TIGR genomic and annotated database [49] resources of rice using the response regulator protein sequences of Arabidopsis thaliana as query. The number and positions of exons and introns for individual OsRR genes were determined by comparison of the cDNAs with their corresponding genomic DNA sequences. The position of each gene on rice chromosomes was found by BLASTN search in genomic sequences of rice chromosome pseudomolecules available at TIGR (Release 3). Multiple sequence alignments were done using the ClustalX (version 1.83) program [50] and the phylogenetic analysis carried out by neighbor-joining method [51]. The unrooted phylogenetic tree was displayed using the Treeview program. The DNA and protein sequence analyses were performed using Gene Runner program version 3.04. Pairwise comparisons were done with the DNASTAR MegAlign 4.03 package to determine the sequence identities.

\section{Plant material and growth conditions}

Rice (Oryza sativa L. ssp. indica var. Pusa Basmati 1) seeds were disinfected with $0.1 \% \mathrm{HgCl}_{2}$ solution for $1 \mathrm{~h}$ and thoroughly washed with RO (reverse-osmosis) water before soaking overnight in RO water. Seedlings were grown on cotton saturated with $\mathrm{RO}$ water, at $28 \pm 1^{\circ} \mathrm{C}$, either in complete darkness or in a culture room with a daily photoperiodic cycle of $14 \mathrm{~h}$ light and $10 \mathrm{~h}$ dark. Flowers and mature leaves were collected from rice plants grown in the greenhouse.

\section{Hormone and stress treatments}

For cytokinin treatment, 6-day-old light-grown rice seedlings were transferred to a solution of $50 \mu \mathrm{M}$ benzyl ami- 
Table 3: Primer sequences used for real time PCR expression analysis.

\begin{tabular}{ll}
\hline Gene name & 'Primer sequence \\
\hline OsRRI & 5'-AGGATCAGCAGATGCATGAATG-3' \\
OsRR2 & 5'-GAGACGCTGTACGTCCTTGCTT-3' \\
& 5'-ACGATCTTCTCAAAGCCATCAAG-3' \\
OsRR3 & 5'-TGAGAGGCTTAAGGATGAAATCCT-3' \\
& 5'-CAGGGTTCGATCTCCTCAAGAG-3' \\
OsRR4 & 5'-CGAATTCTCCGACGACATTAGC-3' \\
& 5'-TCTTCTGAGAATGTGCCTGCAA-3' \\
OsRR5 & 5'-GCTTGACAGGTTTCAGGAAGAACT-3' \\
& 5'-ACCGAATGTGAGCATGATTATCA-3' \\
OsRR6 & 5'-CCTTGACCTTCTTCAGGAGTTCATA-3' \\
& 5'-GTCCCCAACGTCAACATGATC-3' \\
OsRR7 & 5'-CACGTTCTCCGACGACATGAT-3' \\
& 5'-TGCTCAAGAAGATCAAGGAATCG-3' \\
OsRR8 & 5'-GGCACGTTCTCTGACGACATTAT-3' \\
& 5'-CCAGACATGACCGGCTATAACC-3' \\
OsRR9/I0 & 5'-AAGCAATTACAACCGGGAGATG-3' \\
& 5'-TCATGAGGACAGCCCAATTTCTA-3' \\
UBQ65 & 5'-TGCAGTAGTCTGTGATGATCAGGTT-3' \\
& 5'-ACCACTTCGACCGCCACTACT-3' \\
& 5'-ACGCCTAAGCCTGCTGGTT-3' \\
\hline
\end{tabular}

nopurine (BAP) and harvested at the indicated times. For treatment with other hormones/compounds, 6-day-old light-grown rice seedlings were transferred to beakers containing solutions of indole-3-acetic acid (IAA, $50 \mu \mathrm{M}$ ), epibrassinolide (Ebr, $10 \mu \mathrm{M}$ ), gibberellic acid (GA3, 50 $\mu \mathrm{M})$, 1-aminocyclopropane-1-carboxylic acid (ACC, 50 $\mu \mathrm{M})$, abscisic acid (ABA, $50 \mu \mathrm{M}$ ) and cycloheximide $(\mathrm{CHX}, 50 \mu \mathrm{M})$ and incubated for for $3 \mathrm{~h}$. The mocktreated seedlings for the respective time intervals served as the control.

For salt and drought stress treatments, 6-day-old lightgrown rice seedlings were transferred to $250 \mathrm{mM} \mathrm{NaCl}$ or $300 \mathrm{mM}$ mannitol for $6 \mathrm{~h}$. For low temperature treatment, the seedlings were kept at $8 \pm 1{ }^{\circ} \mathrm{C}$ for $6 \mathrm{~h}$. The seedlings kept in water for the same duration, and at $28 \pm 1{ }^{\circ} \mathrm{C}$, served as control.

\section{RNA isolation}

Total RNA was extracted using the RNeasy Plant mini kit (Qiagen, Germany). To remove any genomic DNA contamination, the RNA samples were treated with RNasefree DNase I (Qiagen) according to the manufacturer's instructions. For each RNA sample, absorption at $260 \mathrm{~nm}$ was measured and RNA concentration calculated as $\mathrm{A}_{260} \times$ $40(\mu \mathrm{g} / \mathrm{mL}) \times$ dilution factor. The integrity of RNA samples was monitored by agarose gel elecrophoresis.

\section{cDNA isolation, cloning and sequencing}

The coding region of OsRR genes were amplified by RT$\mathrm{PCR}$, using gene-specific primers, from total RNA isolated from light-grown seedlings using Titan One Tube RT-PCR kit (Roche, USA) according to the manufacturer's instructions. After 30 or 35 cycles, the PCR products were examined by gel electrophoresis and EtBr staining. RT-PCR products were cloned into pGEM-T Easy vector (Promega, Madison, WI) as per manufacturer's instructions. Sequencing was carried out using ABI Prism 377 Sequencer (PE Applied Biosystems, USA), with the Thermosequenase Dye Terminator Cycle Sequencing Kit (Amersham, UK).

\section{Quantitative real-time $P C R$ expression analysis}

The transcript levels of type-A OsRR genes in various RNA samples were quantified by real-time PCR analysis employing ABI Prism 7000 Sequence Detection System and software (PE Applied Biosystems, USA) as described previously [52]. Primers were designed by using the primer design software Primer Express 2.0 (PE Applied Biosystems, USA). To ensure that the primers amplify a unique and desired cDNA segment, each pair of primers was checked in the BLAST program in rice genomic sequence available in TIGR database. The primer sequences are listed in Table 3. Primers specific to OsRR9 and 10 could not be designed as more than $99 \%$ identity was found in the coding region and flanking sequences. First strand cDNA was synthesized by reverse transcribing $3 \mu \mathrm{g}$ of total RNA using High Capacity cDNA Archive kit (Applied Biosystems, USA) according to the manufacturer's instructions. Diluted cDNA samples were used as template and mixed with $200 \mathrm{nM}$ of each primer and SYBR Green PCR Master Mix (Applied Biosystems, USA) for real-time PCR analysis. PCR reactions were performed using the following parameters: $2 \mathrm{~min}$ at $50^{\circ} \mathrm{C}, 10 \mathrm{~min}$ at $95^{\circ} \mathrm{C}$, and 40 cycles of $15 \mathrm{~s}$ at $95^{\circ} \mathrm{C}$ and $1 \mathrm{~min}$ at $60^{\circ} \mathrm{C}$ in 96-well optical reaction plates (Applied Biosystems, USA). The identities of the amplicons and the specificity of the reaction were verified by agarose gel electrophoresis and melting curve analysis, respectively. The relative mRNA levels for each of the OsRR genes in different RNA samples were computed with respect to the internal standard, $U B Q 5$, to normalize for variance in the quality of RNA and the amount of input cDNA. At least two different RNA isolations and cDNA syntheses were used for quantification and each cDNA sample subjected to real-time PCR in triplicate. The values presented are the mean of the two biological replicates, each with three technical replicates. The error bars indicate the standard deviation from the mean.

\section{Authors' contributions}

MJ designed and performed all the in silico analysis and wet-lab experiments, and drafted the manuscript. AKT and JPK participated in design of experiments, coordinated the study, and helped to draft the manuscript. All authors read and approved the final manuscript. 


\section{Acknowledgements}

MJ acknowledges the award of Senior Research Fellowship from the Council of Scientific and Industrial Research, New Delhi. We gratefully acknowledge the financial support of Department of Biotechnology, Government of India, and the University Grants Commission, New Delhi.

\section{References}

I. Mok DW, Mok MC: Cytokinin Metabolism and Action. Annu Rev Plant Physiol Plant Mol Biol 2001, 52:89-I 18.

2. Davies PJ: Plant Hormones: Biosynthesis, Signal transduction, Action The Netherlands: Kluwer Academic Press; 2004.

3. Kakimoto T: Perception and signal transduction of cytokinins. Annu Rev Plant Biol 2003, 54:605-627.

4. Oka A, Sakai H, Iwakoshi S: His-Asp phosphorelay signal transduction in higher plants: receptors and response regulators for cytokinin signaling in Arabidopsis thaliana. Genes Genet Syst 2002, 77:383-391.

5. Hutchison CE, Kieber J): Cytokinin signaling in Arabidopsis. Plant Cell 2002:S47-59.

6. Lohrmann J, Harter K: Plant two-component signaling systems and the role of response regulators. Plant Physiol 2002, I 28:363-369.

7. Hwang I, Chen HC, Sheen J: Two-component signal transduction pathways in Arabidopsis. Plant Physiol 2002, I 29:500-5I 5.

8. Heyl A, Schmulling T: Cytokinin signal perception and transduction. Curr Opin Plant Biol 2003, 6:480-488.

9. Grefen C, Harter K: Plant two-component systems: principles, functions, complexity and cross talk. Planta 2004, 2 I 9:733-742.

10. Stock AM, Robinson VL, Goudreau PN: Two-component signal transduction. Annu Rev Biochem 2000, 69:183-2।5.

II. D'Agostino IB, Deruere J, Kieber J]: Characterization of the response of the Arabidopsis response regulator gene family to cytokinin. Plant Physiol 2000, I 24:1706-1717.

12. Sakai H, Aoyama T, Bono H, Oka A: Two-component response regulators from Arabidopsis thaliana contain a putative DNAbinding motif. Plant Cell Physiol 1998, 39:1232-I239.

13. Sakai H, Aoyama T, Oka A: Arabidopsis ARRI and ARR2 response regulators operate as transcriptional activators. Plant J 2000, 24:703-7II.

14. Mason MG, Li J, Mathews DE, Kieber J], Schaller GE: Type-B response regulators display overlapping expression patterns in Arabidopsis. Plant Physiol 2004, 135:927-937.

15. Makino S, Kiba T, Imamura A, Hanaki N, Nakamura A, Suzuki T, Taniguchi M, Ueguchi C, Sugiyama T, Mizuno T: Genes encoding pseudo-response regulators: insight into His-to-Asp phosphorelay and circadian rhythm in Arabidopsis thaliana. Plant Cell Physiol 2000, 4I:791-803.

16. Makino S, Matsushika A, Kojima M, Yamashino T, Mizuno T: The APRRI/TOCI quintet implicated in circadian rhythms of Arabidopsis thaliana: I. Characterization with APRR I-overexpressing plants. Plant Cell Physiol 2002, 43:58-69.

17. Matsushika A, Makino S, Kojima M, Yamashino T, Mizuno T: The APRRI/TOCI quintet implicated in circadian rhythms of Arabidopsis thaliana: II. Characterization with CCAI-overexpressing plants. Plant Cell Physiol 2002, 43: I I 8-I 22.

18. Murakami M, Ashikari M, Miura K, Yamashino T, Mizuno T: The evolutionarily conserved OsPRR quintet: rice pseudo-response regulators implicated in circadian rhythm. Plant Cell Physiol 2003, 44: $1229-1236$

19. Taniguchi M, Kiba T, Sakakibara H, Ueguchi C, Mizuno T, Sugiyama T: Expression of Arabidopsis response regulator homologs is induced by cytokinins and nitrate. FEBS Lett 1998, 429:259-262.

20. Kiba T, Taniguchi M, Imamura A, Ueguchi C, Mizuno T, Sugiyama T: Differential expression of genes for response regulators in response to cytokinins and nitrate in Arabidopsis thaliana. Plant Cell Physiol 1999, 40:767-77I.

21. Hwang I, Sheen J: Two-component circuitry in Arabidopsis cytokinin signal transduction. Nature 200I, 4I3:383-389.

22. Sakai H, Honma T, Aoyama T, Sato S, Kato T, Tabata S, Oka A: ARRI, a transcription factor for genes immediately responsive to cytokinins. Science 200I, 294:I5I9-I52I.

23. Kiba T, Yamada H, Sato S, Kato T, Tabata S, Yamashino T, Mizuno T: The type-A response regulator, ARRI5, acts as a negative regulator in the cytokinin-mediated signal transduction in Arabidopsis thaliana. Plant Cell Physiol 2003, 44:868-874.
24. To JP, Haberer G, Ferreira FJ, Deruere J, Mason MG, Schaller GE, Alonso JM, Ecker JR, Kieber JJ: Type-A Arabidopsis response regulators are partially redundant negative regulators of cytokinin signaling. Plant Cell 2004, I 6:658-67I.

25. Osakabe Y, Miyata S, Urao T, Seki M, Shinozaki K, Shinozaki KY: Overexpression of Arabidopsis response regulators, ARR4/ ATRRI/IBC7 and ARR8/ATRR3, alters cytokinin responses differentially in the shoot and in callus formation. Biochem Biophys Res Commun 2002, 293:806-8I5.

26. Sweere U, Eichenberg K, Lohrmann J, Mira-Rodado V, Baurle I, Kudla J, Nagy F, Schafer E, Harter K: Interaction of the response regulator ARR4 with phytochrome $B$ in modulating red light signaling. Science 200I, 294: I I08-IIIII.

27. Asakura Y, Hagino T, Ohta Y, Aoki K, Yonekura-Sakakibara K, Deji A, Yamaya T, Sugiyama T, Sakakibara H: Molecular characterization of His-Asp phosphorelay signaling factors in maize leaves: implications of the signal divergence by cytokinin-inducible response regulators in the cytosol and the nuclei. Plant $\mathrm{Mol} B i o$ 2003, 52:33|-34|

28. Giulini A, Wang J, Jackson D: Control of phyllotaxy by the cytokinin-inducible response regulator homologue $A B P H Y L I$. Nature 2004, 430:1031-1034.

29. Goff SA, Ricke D, Lan TH, Presting G, Wang R, Dunn M, Glazebrook J, Sessions A, Oeller P, Varma H, Hadley D, Hutchison D, Martin C, Katagiri F, Lange BM, Moughamer T, Xia Y, Budworth P, Zhong J, Miguel T, Paszkowski U, Zhang S, Colbert M, Sun WL, Chen L, Cooper B, Park S, Wood TC, Mao L, Quail P, Wing R, Dean R, Yu Y, Zharkikh A, Shen R, Sahasrabudhe S, Thomas A, Cannings R, Gutin A, Pruss D, Reid J, Tavtigian S, Mitchell J, Eldredge G, Scholl T, Miller RM, Bhatnagar S, Adey N, Rubano T, Tusneem N, Robinson R, Feldhaus J, Macalma $T$, Oliphant $A$, Briggs $S$ : A draft sequence of the rice genome (Oryza sativa L. ssp. japonica). Science 2002, 296:92-100

30. Yu J, Hu S, Wang J, Wong GK, Li S, Liu B, Deng Y, Dai L, Zhou Y, Zhang X, Cao M, Liu J, Sun J, Tang J, Chen Y, Huang X, Lin W, Ye C, Tong W, Cong L, Geng J, Han Y, Li L, Li W, Hu G, Huang X, Li W, Li J, Liu Z, Li L, Liu J, Qi Q, Liu J, Li L, Li T, Wang X, Lu H, Wu T, Zhu M, Ni P, Han H, Dong W, Ren X, Feng X, Cui P, Li X, Wang H, Xu X, Zhai W, Xu Z, Zhang J, He S, Zhang J, Xu J, Zhang K, Zheng X, Dong J, Zeng W, Tao L, Ye J, Tan J, Ren X, Chen X, He J, Liu D, Tian W, Tian C, Xia H, Bao Q, Li G, Gao H, Cao T, Wang J, Zhao W, Li P, Chen W, Wang X, Zhang Y, Hu J, Wang J, Liu S, Yang J, Zhang G, Xiong Y, Li Z, Mao L, Zhou C, Zhu Z, Chen R, Hao B, Zheng W, Chen S, Guo W, Li G, Liu S, Tao M, Wang J, Zhu L, Yuan L, Yang H: A draft sequence of the rice genome (Oryza sativa L. ssp. indica). Science 2002, 296:79-92.

31. Kolkman JA, Stemmer WPC: Directed evolution of proteins by exon shuffling. Nature Biotech 200I, 19:423-428.

32. Paterson $\mathrm{AH}$, Bowers JE, Chapman BA: Ancient polyploidization predating divergence of the cereals, and its consequences for comparative genomics. Proc Natl Acad Sci USA 2004, | 0 | :9903-9908

33. The Rice Chromosomes II and 12 Sequencing Consortia: The sequence of rice chromosomes II and I2, rich in disease resistance genes and recent duplications. BMC Biology 2005, 3:20.

34. Imamura A, Hanaki N, Umeda H, Nakamura A, Suzuki T, Ueguchi C, Mizuno T: Response regulators implicated in His-to-Asp phosphotransfer signaling in Arabidopsis. Proc Natl Acad Sci USA I998, 95:2691-2696.

35. Imamura A, Yoshino Y, Mizuno T: Cellular localization of the signaling components of Arabidopsis His-to-Asp phosphorelay. Biosci Biotechnol Biochem 2001, 65:2II3-2117.

36. International Rice Genome Sequencing Project: The map-based sequence of the rice genome. Nature 2005, 436:793-800.

37. Remington DL, Vision TJ, Guilfoyle TJ, Reed JW: Contrasting modes of diversification in the Aux/IAA and ARF gene families. Plant Physiol 2004, I 35: I738-I752.

38. Jain M, Kaur N, Garg R, Thakur JK, Tyagi AK, Khurana JP: Structure and expression analysis of early auxin-responsive Aux/IAA gene family in rice (Oryza sativa). Funct Integr Genomics 2006, 6:47-59.

39. Blanc G, Wolfe $\mathrm{KH}$ : Functional divergence of duplicated genes formed by polyploidy during Arabidopsis evolution. Plant Cell 2004, 16:1679-1691. 
40. Rashotte AM, Carson SDB, To JPC, Kieber J]: Expression profiling of cytokinin action in Arabidopsis. Plant Physiol 2003, 132:1998-20II.

4I. Abel S, Nguyen MD, Theologis A: The PS-IAA4/5-like family of early auxin-inducible mRNAs in Arabidopsis thaliana. J Mol Biol 1995, 25 I:533-549.

42. Brandstatter I, Kieber J]: Two genes with similarity to bacterial response regulators are rapidly and specifically induced by cytokinin in Arabidopsis. Plant Cell 1998, 10:1009-1019.

43. Urao T, Yakubov B, Yamaguchi-Shinozaki K, Shinozaki K: Stressresponsive expression of genes for two-component response regulator-like proteins in Arabidopsis thaliana. FEBS Lett 1998, 427: $175-178$.

44. Urao T, Yakubov B, Satoh R, Yamaguchi-Shinozaki K, Seki M, Hiryama T, Shinozaki K: A transmembrane hybrid-type histidine kinase in Arabidopsis functions as an osmosensor. Plant Cell 1999, I I:1743-1754.

45. Halliday KJ, Fankhauser C: Phytochrome-hormonal signalling networks. New Phytol 2003, 157:449-463.

46. Rice Tos 17 Insertion Mutant Database [http:// tos.nias.affrc.go.jp/]

47. Miyao A, Tanaka K, Murata K, Sawaki H, Takeda S, Abe K, Shinozuka $\mathrm{Y}$, Onosato $\mathrm{K}$, Hirochika $\mathrm{H}$ : Target site specificity of the Tos $I 7$ retrotransposon shows a preference for insertion within genes and against insertion in retrotransposon-rich regions of the genome. Plant Cell 2003, I5:177|-1780.

48. National Centre for Biotechnology Information [http:// www.ncbi.nlm.nih.gov]

49. TIGR Rice Genome Annotation [http://tigrblast.tigr.org/eukblast]

50. Thompson JD, Gibson TJ, Plewniak F, Jeanmougin F, Higgins DG: The CLUSTAL_X windows interface: flexible strategies for multiple sequence alignment aided by quality analysis tools. Nucleic Acids Res 1997, 25:4876-4882.

51. Saitou N, Nei M: The neighbor-joining method: a new method for reconstructing phylogenetic trees. Mol Biol Evol 1987 4:406-425.

52. Jain $M$, Kaur N, Tyagi AK, Khurana JP: The auxin-responsive GH3 gene family in rice (Oryza sativa). Funct Integr Genomics 2006, 6:36-46.

Publish with Bio Med Central and every scientist can read your work free of charge

"BioMed Central will be the most significant development for disseminating the results of biomedical research in our lifetime. "

Sir Paul Nurse, Cancer Research UK

Your research papers will be:

- available free of charge to the entire biomedical community

- peer reviewed and published immediately upon acceptance

- cited in PubMed and archived on PubMed Central

- yours - you keep the copyright

Submit your manuscript here:

http://www.biomedcentral.com/info/publishing_adv.asp
BioMedcentral 